\title{
Advanced MR Imaging Techniques in The Diagnosis of Epilepsy
}

\author{
Emad AbdulAzem Tiba ${ }^{\text {a }}$, Medhat Refaat ${ }^{\text {a }}$, Medhat El-sherbiny ${ }^{\text {a }}$, Sameh Abdelaziz Aly
}

aepartment of radiology,
Benha faculty of medicine,
Benha university, Egypt
correspondence to: Emad Abul
Azim Tiba, Department of
radiology, Benha faculty of
medicine, Benha university,
Egypt
email:
emadteba2007@gmail.com

Received: 7 July 2019

Accepted: 14 October 2019

\begin{abstract}
:
Background: Once patients have a diagnosis of localization related epilepsy (LRE), it is critical to further classify those patients into lesional or non-lesional for treatment and prognostic reasons. The cornerstone of lesion detection in chronic epilepsy is structural imaging, mainly magnetic resonance imaging. Molecular \& Metabolic information from Diffusion weighted MRI \& magnetic resonance spectroscopy (MRS) might serve as an additional or as a surrogate marker for the epileptogenic lesion.
\end{abstract}

Methods: 70 patients presented to Aljahra general hospital, Jahra city, Kuwait and were referred to radiology department for MRI study patients represented spectrum for different causes of epilepsy \& different age groups All patients was scanned using Semens Aera 1.5 tesla \& skyra 3 tesla magnets by conventional MRI ((T1WI, T2WI, FALIR), Diffusion weight images ( DWI/ADC) sequences \& MRS were done for patients with SOL, suspected vascular or inflammatory causes. The patients with fits with no MRI signal or structural abnormality are excluded from this study.

Results: DWI \& MRS can provide additional diagnostic information that may facilitate and support the final diagnosis, especially if clinical symptoms are inconclusive. While conventional imaging provided only anatomical information,

Conclusion: Diffusion weighted images (DWI) \& SWI with conventional MRI increased accuracy of diagnosis of lesional epilepsy. 


\section{1- Introduction:}

Epilepsy is a disorder of the brain characterized by an enduring predisposition to generate epileptic seizures and by the neurobiologic, cognitive, psychological, and social consequences of this condition. The definition of epilepsy requires the occurrence of at least one epileptic seizure. However, in contrast to former classifications, one seizure permits the -diagnosis of epilepsy if paraclinical EEG (e.g., $3 \mathrm{~Hz}$ spike and-wave discharges) or MRI (e.g., hippocampal sclerosis) findings point to an increased epileptogenicity. ${ }^{(1)}$

Once patients have a diagnosis of localization related epilepsy (LRE), it is critical to further classify those patients into lesional or nonlesional for treatment and prognostic reasons. An individual with LRE may be classified as nonlesional for two reasons: 1) a lesion may not exist; that is, the structural abnormality that gives rise to seizures may be at the channel level or be spatially distributed in such a way that it would not be accurately termed a lesion, or 2) a lesion exists but is so subtle that standard clinical imaging is not sensitive enough to discriminate between the lesion and surrounding healthy brain tissue. As with any technology and disease process, this definition is dynamic, as we know that future imaging techniques will be developed and new disease mechanisms will be discovered, making detection of the epileptogenic underlying abnormality an everchanging target. ${ }^{(2)}$

Multimodal imaging and future developments of neuroimaging techniques improve our understanding of the dynamics of brain with high spatial and temporal resolutions. The detection of subtle structural or functional abnormalities allow considering surgery in a greater number of difficult cases. Imaging findings have a role in guiding the implantation of intracranial electrodes, for improving the success of subsequent surgery. Hopefully, more patients will benefit from surgery without the need for invasive recordings. The concordance between different imaging techniques facilitates better mapping of epileptic zone, epileptic networks, and eloquent cortices. The understanding of functional brain networks will allow us to better understand the neurobiology of epilepsies and develop new diagnostic, prognostic and therapeutic tools. ${ }^{(3)}$

The cornerstone of lesion detection in chronic epilepsy is structural imaging, mainly magnetic resonance imaging. Metabolic information from magnetic resonance spectroscopy (MRS) might serve as an additional or as a surrogate marker for the epileptogenic lesion. ${ }^{(4)}$

Magnetic resonance spectroscopy (MRS) measures the concentrations of metabolites in 
the brain noninvasively. In epilepsy, MRS aims to aid the identification of the epileptogenic lesion. Ultimately it aims to predict the postoperative outcome after surgical removal of these lesions. ${ }^{(4)}$

Changes on standard diffusion-weighted MRI (DWI) are well established in the ictal and postictal states with restricted diffusion profiles seen in the region of the ictal-onset zone. This is thought to be related to cytotoxic edema being produced secondary to cellular membrane dysfunction and localized cerebral ischemia. Diffusion-tensor imaging (DTI) is a specialized DWI technique that visualizes the movement of water molecules along white matter fibers, thereby allowing visualization of these tracts. It is also possible to retrieve information about the directionality and magnitude of the diffusing water molecules, yielding additional information about the strength of white matter connectivity between brain regions. ${ }^{(5)}$

The aim of our study is to evaluate the current role of some advanced MR Imaging Techniques in the Diagnosis of cases of epilepsy .

\section{2- Patient and Method}

This retrospective study was conducted upon flow of 70 patients presented to Aljahra general hospital, Jahra city, Kuwait. In a three years prospective study from 2016- to 2019. We selected 70 patients from the patients was complaining of epilepsy \& were referred to radiology department for MRI study to assess the presence of epileptogenic lesion after the patient diagnosed by clinical \& EEG examinations as having seizures of different patterns. These 70 patient represented spectrum for different causes of epilepsy \& different age groups.

These procedures are approved by local Ethics Committee. All patients provided a written informed consent.

The patients were selected from different age groups according to some Inclusion \& exclusion criteria as followed;

Inclusion criteria; was Patients presented by fits, Patient complaint proved clinically ( examination \& EEG), and The presence of signal and or structural abnormalities by MRI study. Exclusion criteria; was No H/O of or new presentation of fits, The patients is not assessed or proved clinically, and Apparently normal MRI with no signal or structural abnormalities.

All patients was scanned in radiology department, MRI unite using semens Aera 1.5 tesla \& skyra 3 tesla magnets. Conventional MRI ((T1WI, T2WI, FALIR) was done for all patients which depicted signal and/or structural abnormalities in most cases, then IV contrast given to cases of suspected SOL, vascular abnormalities \& suspected inflammatory causes. Then we applied some 
advanced MRI sequences to the cases according to the type of pathological entity to study their effect on increasing the accuracy of the diagnosis. Diffusion weight images ( DWI/ADC) sequences were done for patients with SOL, suspected vascular or inflammatory causes.

The patients with fits with no MRI signal or structural abnormality are excluded from this study. We selected the patients that revealed signal and or structural abnormalities of different age groups (tables from1to 7).

Table 1: Demographic and Clinical Characters of the Studied Group :

\begin{tabular}{lcc}
$\begin{array}{l}\text { Demographic and } \\
\text { General character }\end{array}$ & $\begin{array}{c}\text { No of cases } \\
\text { Total }=70\end{array}$ & \% \\
\hline Sex & & \\
Male & 39 & 55.7 \\
female & 31 & 44.3 \\
Age & & \\
<18 years & 30 & 42.9 \\
$\begin{array}{l}\text { 18-59 years } \\
\text { >59 years }\end{array}$ & 36 & 51.4 \\
Site of the & 4 & 5.7 \\
epileptogeniclesion & & \\
- intra-axial & & \\
temporal & & \\
- intraxial & 21 & \\
extratempral & & \\
- extra-axial & 47 & \\
\hline
\end{tabular}

Table 2: classification of pathological groups of epilepsy:

\begin{tabular}{|c|c|c|c|}
\hline $\begin{array}{l}\text { Pathological } \\
\text { epileptogenic } \\
\text { lesions }\end{array}$ & $\begin{array}{l}\text { No of cases } \\
\text { Total }=70\end{array}$ & $\%$ & Subdevisions \\
\hline Neoplastic & 20 cases & $(28.6 \%)$ & $\begin{array}{l}\text { Primary low } \\
\text { grade } \\
\text { tumors }=10 \\
\text { Primary high } \\
\text { grade tumors=7 } \\
\text { Secondary } \\
\text { ( mets.) } \\
\text { tumors=3 }\end{array}$ \\
\hline Vascular & 12 cases & $(15.7 \%)$ & $\begin{array}{l}\text { Ischemic }=8 \\
\text { Non- } \\
\text { ischemic }=4\end{array}$ \\
\hline $\begin{array}{l}\text { Congenital \& } \\
\text { developmental } \\
\text { Inflammatory }\end{array}$ & $\begin{array}{l}24 \text { cases } \\
14 \text { cases }\end{array}$ & $\begin{array}{l}(34.3 \%) \\
(21.4 \%)\end{array}$ & \\
\hline
\end{tabular}

Table 3: classification of studied patients regarding their presentation:

\begin{tabular}{lcc}
\hline $\begin{array}{l}\text { Pathological epileptogenic } \\
\text { lesions }\end{array}$ & $\begin{array}{l}\text { No of cases } \\
\text { Total = 70 }\end{array}$ & $\%$ \\
\hline Fits/ seizures only. & 28 cases & $(40 \%)$ \\
$\begin{array}{l}\text { Fists/ seizures pulse other } \\
\text { presentations ( headache, } \\
\text { vomiting, behavioral } \\
\text { changes \& fever) }\end{array}$ & 42 cases & $(60 \%)$ \\
\hline
\end{tabular}

Table 4: Finding of Convential MRI of the studied cases of epilepsy $\quad($ no=70 cases $)$ :

\begin{tabular}{lll}
\hline Convential MRI & No & $(\%)$ \\
\hline & & \\
- Diagnostic & 59 & $(84.3)$ \\
-Not diagnostic & 11 & $(15.7)$ \\
& & \\
\hline
\end{tabular}


Table 5: Effect of contrast to differentiate neoplastic and Non neoplastic pathological lesion (38 cases):

\begin{tabular}{|c|c|c|c|c|c|}
\hline & \multicolumn{2}{|c|}{ Pathological lesion } & \multirow{2}{*}{ Total } & \multirow{2}{*}{$\begin{array}{r}\text { Test of significant } \\
\mathbf{X}^{2}\end{array}$} & \multirow{2}{*}{$P$ value } \\
\hline & Neoplastic & Non neoplastic & & & \\
\hline & $(N=19)$ & $(\mathrm{N}=19)$ & $(\mathrm{N}=38)$ & & \\
\hline & No $(\%)$ & No $(\%)$ & No $(\%)$ & & \\
\hline $\begin{array}{l}\text { Contrast } \\
\text { Enhanced }\end{array}$ & $13(68.4)$ & $11(57.9)$ & $24(63.2)$ & 1.452 & 0.369 \\
\hline Not Enhanced & $6 \quad(31.6)$ & $8 \quad(42.1)$ & $14(36.8)$ & & \\
\hline
\end{tabular}

Table 6 : Accuracy of DWI in predicting different types of pathology

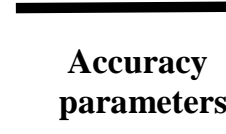
parameters
Type of lesion
Neoplastic

\section{Vascular}

20

(15.39- 59.22)

72

$(50.61-87.83)$

Specificity \%

(95\% CI)

Positive predictive values $\%$ (95\% CI)

Negative predictive values $\%$ (95\% CI)

Like hood ratio of positive results \% (95\% CI)

Like hood ratio of negative results \% (95\% CI)

\section{Accuracy}

(95\% CI)

Area Under Curve

(95\% CI)

$P$ value
50

(29.64-60)

58.06

(48.04-67.47)

1.25

(0.53- 2.97)

$$
\begin{gathered}
0.90 \\
(0.6-1.35)
\end{gathered}
$$

\subsection{6}

(48.04-67.47)

0.54

(0.356- 0.724)
(2.52- 55.61)

65.71

(47.79-80.87)

14.29

(4.26-38.46)

74.19

(66.03-80.96)

0.58

(0.16-2.19)

1.22

(0.82-1.8)

55.56

(40- 70.36)

0.442

( 0.264-0.621)

0.542
Inflammatory
(41.9- 91.61)

70.97

(51.96-85.78)

35.71

(18.54-57.56)

70.97

(60.9 - 79.32)

1.23

(0.5-3)

0.91

(0.58-1.42)

60 $(44.33-74.3)$

0.533

(0.356- 0.724)

0.722 
Table 7: Accuracy of MRS in predicting neoplastic and inflammatory

\begin{tabular}{|c|c|c|}
\hline $\begin{array}{l}\text { Accuracy } \\
\text { parameters }\end{array}$ & Neoplastic & Inflammatory \\
\hline $\begin{array}{l}\text { Sensitivity \% } \\
(95 \% \text { CI }\end{array}$ & $\begin{array}{c}75 \\
(50.91-91.34)\end{array}$ & $\begin{array}{c}57.14 \\
(28.86-82.34)\end{array}$ \\
\hline $\begin{array}{l}\text { Specificity \% } \\
(95 \% \text { CI })\end{array}$ & $\begin{array}{c}42.86 \\
(17.66-71.14)\end{array}$ & $\begin{array}{c}25 \\
(8.66-49.1)\end{array}$ \\
\hline $\begin{array}{l}\text { Positive predictive values } \% \\
(95 \% \text { CI })\end{array}$ & $\begin{array}{c}65.22 \\
(52.73-75.92)\end{array}$ & $\begin{array}{c}34.78 \\
(24.08-47.27)\end{array}$ \\
\hline $\begin{array}{l}\text { Negative predictive values \% } \\
(95 \% \text { CI })\end{array}$ & $\begin{array}{c}54.55 \\
(48.04-67.47)\end{array}$ & $\begin{array}{c}45.45 \\
(24.00-68.75)\end{array}$ \\
\hline $\begin{array}{l}\text { Like hood ratio of positive results \% } \\
(95 \% \mathrm{CI})\end{array}$ & $\begin{array}{c}1.31 \\
(0.78-2.21)\end{array}$ & $\begin{array}{c}0.76 \\
(0.54-1.28)\end{array}$ \\
\hline $\begin{array}{l}\text { Like hood ratio of negative results \% } \\
(95 \% \mathrm{CI})\end{array}$ & $\begin{array}{c}0.58 \\
(0.22-1.54)\end{array}$ & $\begin{array}{c}1.71 \\
(0.65-4.53)\end{array}$ \\
\hline $\begin{array}{l}\text { Accuracy } \\
\text { (95\% CI) } \\
\text { Area Under Curve } \\
(95 \% \text { CI }) \\
\text { P value }\end{array}$ & $\begin{array}{c}61.76 \\
(43.57-77.83) \\
0.599 \\
(0.391-0.807) \\
0.357\end{array}$ & $\begin{array}{c}38.24 \\
(22.17-56.44) \\
0.401 \\
(0.193-0.609) \\
0.357\end{array}$ \\
\hline
\end{tabular}

\section{3- Discussion:}

Neoplastic lesions as a cause of epilepsy represented the largest group in our study it consisted of 10 cases of low grade primary tumors that involved 4 cases of low grade glioma , 3 cases of oligodendroglioma, 1 case of DENT, 1 case of ganglioglioma \& 1 case diagnosed as gliomatosis cerebri. The high grade tumours in our study was 7 cases involved; 1 case of anaplastic oligodendroglioma, 2 cases are high grade gliomas \&4 cases diagnosed as glioblastoma multiforms. The secondary brain tumours that presented with fits \& seizures were three cases. The ratios in our study even with the much difference in the number of patients are to some degree in agreement with a study done by ( Lote et al,1998) for an adult series of 1028 gliomas which postulated that seizures occurred in $49 \%$ of patients with glioblastoma, in $69 \%$ of patients with 
anaplastic glioma, and in $85 \%$ of patients with low-grade glioma .

Infections of the CNS are a major risk factor for epilepsy. The reported risk of unprovoked seizures in population-based cohorts of survivors of CNS infections from developed countries is between 6.8 and $8.3 \%$, and is much higher in resource-poor countries. ${ }^{(6)}$

Infections may also induce status epilepticus, defined as one continuous, unremitting seizure lasting longer than $5 \mathrm{~min}$, or recurrent seizures without the person returning to a normal state between them. ${ }^{(7)}$

Seizures may be induced by brain alterations in response to neurotropic infectious agents that target the CNS (which may include immune/ inflammatory-mediated responses intrinsic to the infected brain tissue). They may result from immune responses to systemic (non-CNS) infections resulting in pro-inflammatory cytokine-induced alterations in BBB integrity and subsequent neuronal hyperexcitability. ${ }^{(8)}$

A wide variety of CNS infections, including bacterial (e.g. typical bacterial meningitis, tuberculosis), viral (e.g. herpes simplex, HHV-6), parasitoses (e.g. cerebral toxoplasmosis, NCC, malaria), fungal (e.g. candidiasis, coccidioidomycosis, aspergillosis), and prion infections (CJD), can lead to status epilepticus. When status epilepticus occurs with encephalitis it has a worse prognosis than those due to other aetiologies . Autoimmune encephalitis is increasingly recognized as a cause of status epilepticus where no infectious organism is identified. $^{(9)}$

It is important to differentiate between early seizures, which may occur within the first 1-2 weeks after infection and late unprovoked seizures, which occur later (often months to years) after infection and are therefore defined as acquired epilepsy. ${ }^{(10)}$

In our study there are 14 cases presented with epilepsy secondary to infective \& inflammatory etiologies as follows; 4 cases of NCC , 4 cases of tuberculomas \& tuberculous meningitis, 3 cases of encephalitis ( herpitic , non-herpitic \& autoimmue), A case of Russmosen encephalitis, a case of ADEM (acute disseminating encephalomyelitis) \& a case of subdural empyema.

Neurocysticercosis (NCC) is a common neurological infestation and probably the major risk factor for acquired epilepsy in many African, Asian and Latin American countries. It is estimated that $30-50 \%$ of cases of epilepsy in endemic regions have NCC as a risk factor (Garcia et al, 2014). In our study the chosed 4 cases are from Asian race ( indians).

CNS tuberculosis has various imaging appearances, including meningitis, tuberculoma, miliary tuberculosis, abscess, 
cerebritis, and encephalopathy. In addition, the radiologic manifestations of this disease are not always typical and sometimes may be mistaken with other lesions such as brain tumors. $^{(11)}$

In our study there is 4 cases of epilepsy diagnosed by clinical,laboratory \& imaging findings as cerebral tuberculosis in the form of tuberculomas in two cases, one case of tubercular abscess \& associated tubercular meningitis is also seen. Encephalitis with prominent epileptic seizures is an antibodyassociated disease reported in patients with full-blown brain neuropathology and in association with cryptogenic epilepsy (e.g., normal brain MRI). ${ }^{(12)}$

Viral encephalitis by definition is the result of human virus affecting the brain and sparing the meninges. The other nervous system manifestations are meningitis, meningoencephalitis, encephalomyelitis, and encephalomyeloradiculitis. Encephalitis can involve any age group from children to old people. The severity of the disease depends on the viral agent and the host immune system. The patient can present with fever, headache, seizure, neurological deficit, or altered sensorium. Laboratory investigations, imaging, and cerebrospinal fluid analysis are crucial in the diagnosis of encephalitis. Magnetic resonance imaging (MRI) findings may be nonspecific or specific and plays a major role in the diagnosis of encephalitis and predicting the possible cause. ${ }^{(13)}$

Autoimmune encephalitis is a relatively new category of immune-mediated disease involving the central nervous system that demonstrates a widely variable spectrum of clinical presentations, ranging from the relatively mild or insidious onset of cognitive impairment to more complex forms of encephalopathy with refractory seizure. Due to its diverse clinical features, which can mimic a variety of other pathologic processes, autoimmune encephalitis presents a diagnostic challenge to clinicians. Imaging findings in patients with these disorders can also be quite variable, but recognizing characteristic findings within limbic structures suggestive of autoimmune encephalitis can be a key step in alerting clinicians to the potential diagnosis and ensuring a prompt and appropriate clinical work-up. ${ }^{(14)}$

Rasmussen's encephalitis is a devastating syndrome of multifocal brain dysfunction and focal seizures. Magnetic resonance (MR) findings, associated with clinical data and electroencephalogram (EEG), may indicate the diagnosis and could be an indicative of prognosis. Andreia et al studied 5 patients with Rasmussen's encephalitis, assessing clinical history and MR images. All patients had refractory focal seizures with a predominant motor component associated 
with hemispheric atrophy that was proportional to severity of disease and neurological deficits in these patients. Gray and white matter abnormal signal on T2 MR Images were found in patients who had hemiparesis. It was not related to the duration of the disease but to aggressiveness. MR proton spectroscopy in severe disease showed lactate and choline increase and decreased NAA, reflecting neuronal and axonal loss, gliosis and elevated membrane. ${ }^{(15)}$

In our study there was a case of Rasmussen encephalitis which seen in 2 y female child that revealed history of perinatal asphyxia \& presented with right hemiparesis, seizures, status epilepticus \& developmental delay.

Pillars et al did cohort study of 164 children with acute encephalitis, and found the larger causes of encephalitis (in descending order) to be: ADEM $(n=35,21 \%)$, enterovirus $(\mathrm{n}=20,12 \%)$, M. pneumoniae $(\mathrm{n}=11, \quad 7 \%)$, NMDAR antibody $(\mathrm{n}=10,6 \%)$, and HSV $(\mathrm{n}=9,5 \%)$. There were 46 patients $(28 \%)$ without an identified etiology (unknown). ${ }^{(16)}$

In our study the vascular causes represented 12 cases $(15.7 \%)$, ischemic related epilepsy are noted in 8 cases $\&$ the other 4 cases are space occupying lesions of vascular origin. The increased prevalence of post stroke seizers \& epilepsy is in agreement with the study done by Jiun-Chang et al. ${ }^{(17)}$
Jiun-Chang et $\mathrm{al}^{(17)}$ did retrospective study, collected clinical and radiographic data on children (age range, 1 month to 18 years) with symptoms and radiographic confirmation of seizure after ischemic stroke for the period of January 1996 to July 2006. Thirty-nine out of 94 children with ischemic stroke had poststroke seizures. Thirty-three out of 39 children with poststroke seizures had new onset seizures but only data of 28 were available. The study concluded that seizures commonly occur in childhood ischemic stroke. Most poststroke seizures developed at an early stage. Infection was the most common etiology that caused early poststroke seizures in childhood ischemic stroke. Poststroke seizures did not affect mortality, but there was a significant difference in normal outcome and epilepsy between those with or without poststroke seizures.

In our study there is 24 cases of epilepsy due to congenital \& developmental causes represented $34.3 \%$ of the studied sample. They are 4cases of neurocutaneous diseases ( phakomatosis ) ( 2 cases of tuberous sclerosis , one case of sturge weber disease $\&$ one case of NF1). 4 cases of mesial temporal sclerosis , 3 cases of focal cortical dysplasia , 2 cases of holoprosencephaly, 2 cases of chizencephaly $\&$ two cases of hetrotopia.

A prospective cohort study conducted over a 2 year period. 102 children of seizure disorders studied by Nita et al revealed that 
out of which $28(27.5 \%)$ Patients were diagnosed to have central nervous system malformations diagnosed by neuroimaging with relevant clinical features. Among those 28 patients of seizure disorder with CNS malformations neural tube defects were the most common (11.76 \%) malformations followed by malformations of cortical development (10.78\%) while neurocutaneous syndromes were less common and isolated dysgenesis Of corpus collasum was least common (1.96\%) malformations. These results are in agreement with that Dolk et al \& Girgis et al. ${ }^{(18)(19)}$

\section{4- References:}

1-Kwan P, Arzimanoglou A, Berg AT, Brodie MJ, Hauser WA, Gary Mathern G, et al (2010):Definition of drug resistant epilepsy: consensus proposal by the ad hoc task force of the ILAE commission on therapeutic strategies. Epilepsia 51(6):1069-1077.

2- Heath P \& Ruben K (2014): Advanced imading techniques in diagnosis of non lesional epilepsy: MRI, MRS, PET \& SPECT, Eplipsy Curr. May-June; 14(3): 121-124.

3-Pittau F, Mégevand P, Sheybani L, Abela E, Grouiller F, Spinelli L, et al (2014): Mapping epileptic activity: sources or networks for the clinicians?, Front Neurol. Nov 5;5:218.

4- Christian G \& Bien (2013): International consensus classification of hippocampal sclerosis in temporal lobe epilepsy: a Task Force report from the ILAE Commission on Diagnostic Methods.
5- Ruben I Kuzniecky (2008): Neuroimaging in epilepsy, Neuroimaging p. 175-187, Vol.14, No.4.

6- Vezzani A, Fujinami RS, White HS, Preux PM, Blümcke I, Sander JW, et al. (2016): Infections, inflammation and epilepsy, Acta Neuropathol ; 131(2): 211-234.

7- Lowenstein DH (2005): Treatment options for status epilepticus. Curr Opin Pharmacol. ; 5:334-339.

8- Marchi N, Granata T, Janigro D (2014): Inflammatory pathways of seizure disorders, Trends Neurosci. ; 37:55-65.

9- Lowenstein DH, Walker M, Waterhouse E (2014): Status epilepticus in the setting of acute encephalitis. Epilepsy Curr. ; 14:43-49.

10-Lowenstein DH (2009): Epilepsy after head injury: an overview. Epilepsia. 50(Suppl 2):4-9.

11- Morteza ST, Mohammad AK, Hamidreza H, Pourghorban R, Samadian M and Kasmaei HD. ( 2015 ): Central Nervous System Tuberculosis: An Imaging-Focused Review of a Reemerging Disease, Radiology Research and Practice.

12- Bien CG, Granata T, Antozzi C, Cross JH, Dulac O, Kurthen M, et al (2005): Pathogenesis, diagnosis and treatment of Rasmussen encephalitis A European consensus statement. Brain.; 128:454471.

13-KalaivaniJ, Rajeswaran $R$ and Anupama C (2018): Magnetic Resonance Imaging Findings in Viral Encephalitis: A Pictorial Essay, J Neurosci Rural Pract ; 9(4): 556-560.

14- Kelley BP, Patel SC, Marin HL, Corrigan JJ, Mitsias PD and Griffith B. (2017): Autoimmune Encephalitis: Pathophysiology and Imaging Review of an Overlooked Diagnosis, American Journal of Neuroradiology, 38 (6) 1070-1078. 
15- Andréia VF, Fabiano R, Guilherme CD (2009): Mri Findings In The Diagnosis And Monitoring Of Rasmussen's Encephalitis, Arq Neuropsiquiatr ;67(3-B):792-797.

16- Pillai SC, Shekeeb SM, Hacohen Y (2016): Postencephalitic epilepsy and drug-resistant epilepsy after infectious and antibody-associated encephalitis in childhood: Clinical and etiologic risk factors, Epilepsia, 57(1):e7-e11.

17- Jiun-Chang L, Kuang L, Huei SW (2009): Seizures in childhood ischemic stroke in
Taiwan,Brain and Development, Volume 31, Issue 4, Pages 294-299.

18- Dolk H, Loane M, Garne E ( 2010): The prevalence of congenital anomalies in Europe. AdvExp Med Biol. 2010;686:349-64.

19- Girgis MY, Mansour LA, Abdullah N, Kamel AF and Antar A (2010): Prospective study on congenital CNS malformations in neuro-pediatric unit Cairo University Egypt. J Neuronal PsychaitNeurosurg 2010;47:275-80.

To cite this article: Emad AbdulAzem Tiba, Medhat Refaat, Medhat El-sherbiny, Sameh Abdelaziz Aly. Advanced MR Imaging Techniques in The Diagnosis of Epilepsy. BMFJ XX DOI: 10.21608/bmfj.2020.14508.1012 